\title{
Modèle mathématique pour la prédiction de la vitesse d'érosion
}

\author{
A. Karimi \\ Institut de génie atomique, Ecole polytechnique fédérale de Lausanne
}

\section{Introduction}

La prédiction de la vitesse d'érosion intéresse en premier lieu les constructeurs de machines hydrauliques en raison de son importance dans la durée de garantie et dans la limitation du design. A cet effet, des efforts considérables ont été faits pour prédire l'érosion et pour corréler la vitesse d'érosion aux propriétés mécaniques ou physicochimiques des matériaux. Des approches théoriques ou des lois empiriques basées sur les méthodes suivantes ont été proposées :

- Au début des problèmes d'érosion, l'idée était de déterminer des relations empiriques ou d'établir des fonctions de corrélation entre la vitesse d'érosion et le comportement mécanique volumique des matériaux comme: dureté, limite d'endurance, résistance à la rupture, etc. [F. G. НАмmitT et al., 1970, F. J. HeYMANN, 1970, P. V. RAO et al., 1981].

- Pour prédire le comportement à l'érosion à long terme, les méthodes de "curve-fitting " ont été largement utilisées. En effet, ce type de travaux consiste d'abord à normaliser les courbes d'érosion obtenues expérimentalement et de lisser ensuite selon des relations mathématiques combinées entre la vitesse instantanée ou accumulée d'érosion avec le temps d'exposition. L'extrapolation de la forme normalisée de ces courbes prédit le comportement à long terme des matériaux à l'attaque de cavitation [F. J. HEYMANN, 1967, P. V. RAO, 1984, A. THIRUVENGADAM, 1967].
- Des techniques électrochimiques, basées sur des mesures du courant ou du potentiel de corrosion entre l'échantillon et une électrode de référence constituant un ensemble électrochimique ont aussi été employées. Ces techniques sont applicables plutôt à des cas d'érosion dans un milieu corrosif et elles semblent avoir donné des résultats satisfaisants concernant la détection du début d'érosion et la simulation de la vitesse d'érosion [L. Chincholle, R. Simoneau]. Dans des milieux moins corrosifs où l'érosion se produit plutôt par des effets mécaniques que par des réactions chimiques, ces techniques ne peuvent être utilisées.

- Application de l'émission acoustique due à la cavitation à la détection du début de la cavitation ou à la simulation du bruit de cavitation avec la vitesse d'érosion. Dans ces méthodes, on propose généralement une relation rationnelle entre la vitesse d'érosion et la puissance acoustique de la cavitation [F. G. НАMMITT, 1979, S. HATTORI et al., 1985]. Le problème fondamental dans ce genre de traitement est de corréler la puissance du son à l'intensité d'impact due à la collapse.

- Il existe également dans la littérature des travaux sur la mesure « in situ " de la vitesse d'érosion usant de techniques physiques comme l'activation des couches minces [D. J. FINNIGAN et al., 1982] ou la dépendance en masse de la fréquence propre des échantillons [F. JANSEN, 1987].

D’une manière générale, ces méthodes n'ont pas donné de résultats satisfaisants pour des larges domaines

\section{Mathematical model predicting cavitation erosion rate.}

A mathematical model incorporating the erosion process and the internal hardening mechanisms has been proposed to predict cavitation erosion rate of alloys. It takes into account both the material properties and the hydrodynamic conditions of cavitation. According to this model, the eroding material is subjected to the stress pulse loading conditions originated from cavitation collapse. The spacial and the temporal distribution of these pulses are random, but their amplitude is controlled by the flow conditions. Beside of mechanical properties, some metallurgical parameters such as work hardening rate and stacking fault energy have been introduced in the model. It can be applied to all types of cavitation and can be extended to erosion by liquid drop impacts or solid particle impact erosion. 
d'application. Les raisons de leur défaillance ont été expliquées dans une autre publication [A. KARIMI, W. LEO, 1987]. En effet, elles ne tiennent pas compte à la fois des propriétés mécaniques des matériaux et des conditions hydrodynamiques du fluide cavitant, c'est-àdire de l'agressivité de cavitation. Le caractère dynamique et pulsatoire du chargement lors de l'érosion ainsi que le caractère localisé de la contrainte et la vitesse élevée de déformation $\left(10^{4}-10^{6} \mathrm{~s}^{-1}\right)$ font que le comportement mécanique des matériaux diffère de celui de la déformation volumique. Par conséquent, les données mécaniques couramment utilisées dans les équations d'érosion doivent correspondre aux conditions d'érosion et non pas aux conditions des essais mécaniques conventionnels.

D'après ces observations, nous avons développé un modèle de prédiction de la vitesse d'érosion décrivant le processus physique d'érosion, incluant à la fois les propriétés mécaniques des matériaux et les conditions hydrodynamiques d'écoulement relatives à la puissance destructive de cavitation. En plus des propriétés mécaniques, nous avons introduit deux paramètres métallurgiques: l'énergie de faute d'empilement et le taux d'écrouissage qui ont une grande influence sur la résistance à l'érosion des alliages.

\section{Base du modèle}

Le but principal de ce modèle est de décrire la vitesse d'érosion comme une fonction explicite du comportement des matériaux et des conditions hydrodynamiques de cavitation. Les études expérimentales sur la résistance à l'érosion des alliages ont conduit à penser que des paramètres métallurgiques comme l'énergie de défaut d'empilement [B. RAO et al., 1984, D. WOODFORD, 1972] et la capacité d'écrouissage [A. BALL, 1983, S. VAIDYA et al., 1980] ont un effet primordial sur la résistance à l'érosion des alliages. La capacité d'écrouissage élevée et l'énergie de défauts d'empilement bas sont des facteurs concomitants dans l'érosion. Il est donc important d'introduire aussi ces paramètres métallurgiques dans la fonction du taux d'érosion.

La première étape dans le développement de notre modèle fut la réalisation des essais d'érosion dans des conditions de cavitation réalistique ou très approchantes de celle-ci afin d'obtenir des courbes d'érosion exactes; car les résultats obtenus sur des dispositifs de cavitation vibratoire ne reflètent pas tout à fait les conditions d'érosion dans des machines [A. KARIMI, F. AvELLAN, 1986]. C'est dans cette optique que nous avons utilisé un générateur de vortex « veine tourbillon " dans lequel le phénomène de cavitation se rapproche de celui rencontré dans les installations hydrodynamiques. L'appareillage et le fonctionnement du dispositif a été décrit ailleurs [F. Avellan, A. Karimi, 1987]. Dans une étude comparative des mécanismes d'érosion dans diverses situations de cavitation érosive, nous avons démontré que le mécanisme d'érosion dans cette machine ressemble à celui observé dans des applications pratiques [A. KARIMI, F. Avellan, 1986]. Durant notre expérience, l'idée était de maintenir stationnaire l'état du fluide et les conditions des essais. Nous avons donc contrôlé la température de travail, la vitesse d'écoulement, la quantité d'air dissoute dans l'eau du circuit et son $\mathrm{pH}$. Pour les essais à long terme, l'eau du circuit a été dégasée périodiquement et régulièrement afin d'éviter la diffusion et la concentration d'air dissous dans la chambre d'essai et par là même l'effet coussin de poche d'air dans le collapse. Les courbes typiques de la perte de masse et de vitesse d'érosion ont une forme simple et monotonique. Sur ces courbes, on ne distingue que deux périodes : accélération et stationnaire. Durant le stade d'accélération, la vitesse d'érosion augmente au fur et à mesure que la surface s'écrouit et que l'érosion s'étend à toute la surface exposée. Dans le stade avancé, l'état de surface ne change pas d'une manière significative et le dégât pénètre dans le volume avec une vitesse moyenne constante. Dans ces conditions, il $\mathrm{y}$ a trois paramètres à calculer : la vitesse d'érosion dans la période stationnaire $\dot{E}$, le temps d'exposition $t_{i}$ correspondant à la fin de la période d'accélération et la vitesse variable d'érosion durant cette période $\dot{e}(t)$.

La vitesse d'érosion, $\dot{e}(t)$, est généralement définie comme la perte instantanée de masse (ou du volume) par unité de temps ou par un nombre défini d'impacts:

$$
\dot{e}(t)=\rho \frac{\mathrm{d} v}{\mathrm{~d} N} \cdot \frac{\mathrm{d} N}{\mathrm{~d} t}
$$

où $N=$ nombre d'impacts dus aux collapses, $t=$ temps, $v=$ volume, $\rho=$ densité du matériau érodé. Pour établir l'équation (1), nous calculons le nombre $N$ d'impacts nécessaires pour déformer le volume $v$ du matériau jusqu'à la rupture $\varepsilon_{R}$. On suppose qu'en cas d'érosion, le matériau est soumis à des pulses de contrainte localisés dont la taille et la distribution spatiale sont statistiques. La variation de leur amplitude est aussi aléatoire mais leur valeur moyenne est contrôlée par les conditions d'écoulement. D'après ces observations, la vitesse d'érosion en un point, $x$, de l'échantillon à l'instant, $t$, peut être écrit :

$$
\dot{e}(x, t)=\rho \frac{\mathrm{d}}{\mathrm{d} N}[S(N) \cdot \eta(h) \cdot \psi(x) \cdot \xi(t)] \frac{\mathrm{d} N}{\mathrm{~d} t}
$$

$S(N)=$ fonction de distribution de la taille des impacts sur la surface,

$\eta(h)=$ facteur de pénétration du dégât dans le volume,

$\psi(x)=$ distribution spatiale des collapses,

$\xi(t)=$ variation temporelle de l'amplitude des pulses en fonction du temps d'exposition.

Les deux premiers termes de l'équation (2) relèvent du comportement du matériau face à l'attaque de cavitation et les deux derniers décrivent les conditions hydrodynamiques et l'agressivité de cavitation. Tous les termes de l'équation (2) sont des fonctions multi-variantes. Par exemple, dans un profil NACA exposé dans un tunnel de cavitation, $\psi(x)$ dépend de la géométrie du bord et présente un pic à l'extrémité de la poche dont l'étendue dépend de l'angle d'attaque, de la vitesse d'écoulement et de la $\sigma$. Cependant, les essais d'érosion en laboratoire sont généralement effectués dans des conditions contrôlées et les fonctions $\psi(x)$ et $\xi(t)$ ont alors des valeurs 
constantes. Quant à la fonction de distribution de la taille des impacts, la connaissance actuelle sur la cavitation ne permet pas de l'estimer théoriquement. Les exemples d'impacts isolés (fig. 1) montrent que pour une même condition de travail, les tailles des impacts dépendent des conditions locales de cavitation et diffèrent sensiblement.

La fonction de pénétration du dégât $\eta(h)$ intègre en effet la déformation locale des impacts isolés dans différents états de durcissement du matériau. Ceci est illustré par un graphique dans la figure 2 où l'écrouissage décroît en s'éloignant du point d'impact. Pour le calcul, le profil de déformation est exprimé par l'équation suivante :

$$
\Delta \varepsilon_{x}=\Delta \varepsilon_{0}(1-x / l)^{\theta}
$$

$\Delta \varepsilon_{0}=$ accroissement de déformation dans la zone d'impact,

$\Delta \varepsilon_{x}=$ accroissement de déformation à une distance $x$ du point d'impact,

$=$ profondeur d'écrouissage,

= paramètre métallurgique traduisant la capacité de durcissement interne des matériaux.

\section{Vitesse d'érosion en stade stationnaire}

Le principe mathématique du calcul est basé sur le processus physique d'érosion. En effet, on suppose que les pulses de pression dus aux collapses de cavité atteignent la surface du matériau d'une manière aléatoire mais en réalité leur distribution spatiale est contrôlée par le champ d'écoulement. Ces pulses écrouissent les couches superficielles du matériau, provoquant des fractures locales et propageant des fissures. Lorsque les couches superficielles sont entièrement écrouies, le dégât pénètre dans le volume des matériaux. La vitesse de l'endommagement durant ce stade est la vitesse d'érosion en la période stationnaire de la courbe d'érosion. Il est à noter que seuls les pulses dont l'amplitude est plus élevée que la contrainte de rupture du matériau exposé, $\sigma_{R}$, provoquent la rupture et la perte de masse. Les pulses se situant entre la limite de rupture et la limite d'élasticité $\sigma_{0}$ déforment et écrouissent la surface et contribuent au durcissement interne. Les pulses inférieures à la limite d'élasticité n'ont pas d'effet significatif dans l'érosion. Les diagrammes de la figure 3 (page suiv.) illustrent les différentes étapes du calcul. En appliquant l'expression de l'équation (3) au profil de déformation dans la zone exposée et en établissant la relation $\sigma-\sigma_{0}=\kappa \varepsilon^{n}$ entre la contrainte appliquée et la déformation, on calcule d'abord l'épaisseur des couches érodées $\Delta h[14]$ comme suit :

$$
\Delta h=\left[\left(\frac{\sigma_{\mathrm{am}}-\sigma_{0}}{\sigma_{R}-\sigma_{0}}\right)^{\frac{1}{n \theta}}-1\right] L
$$

$L \quad=$ profondeur d'écrouissage, tel que montré dans la figure 3 .

$\sigma_{\text {am }}=$ valeur moyenne d'amplitude des pulses se situant au-delà de la limite de rupture.
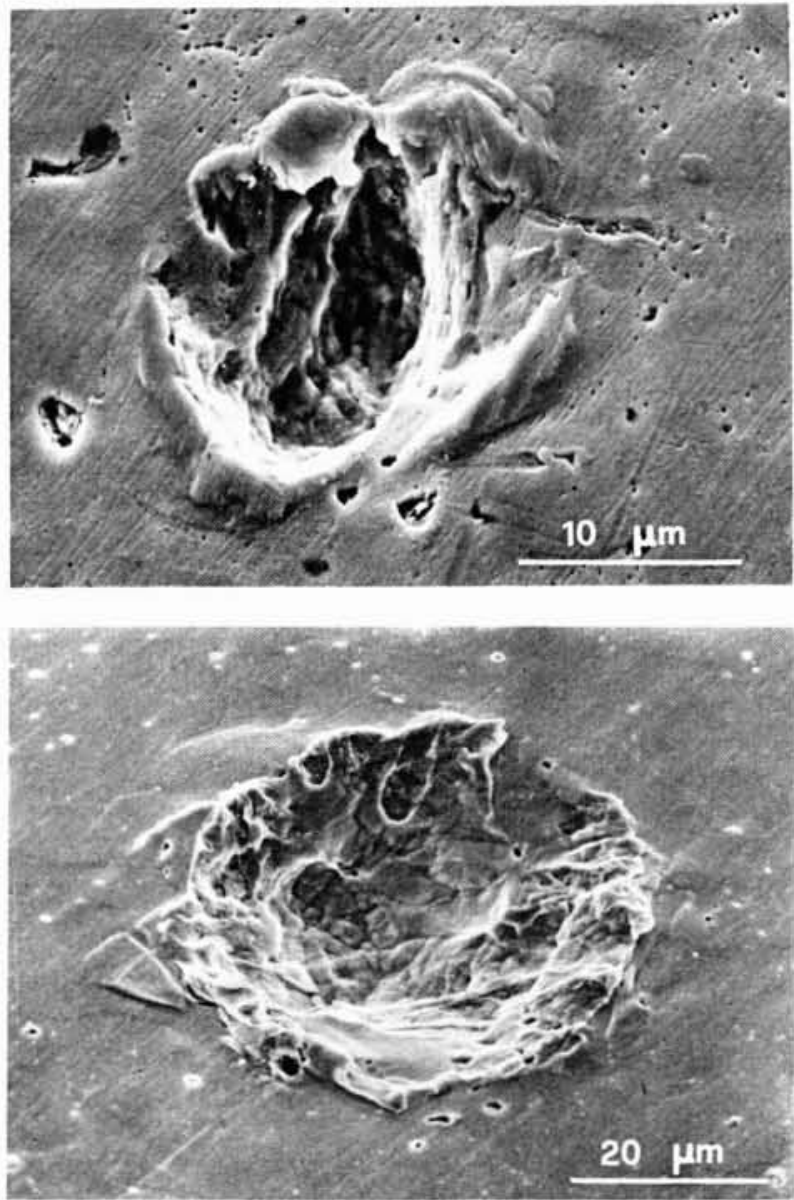

1. Exemples d'impacts isolés sur un laiton $(\alpha+\beta)$ dus aux collapses de cavitation.

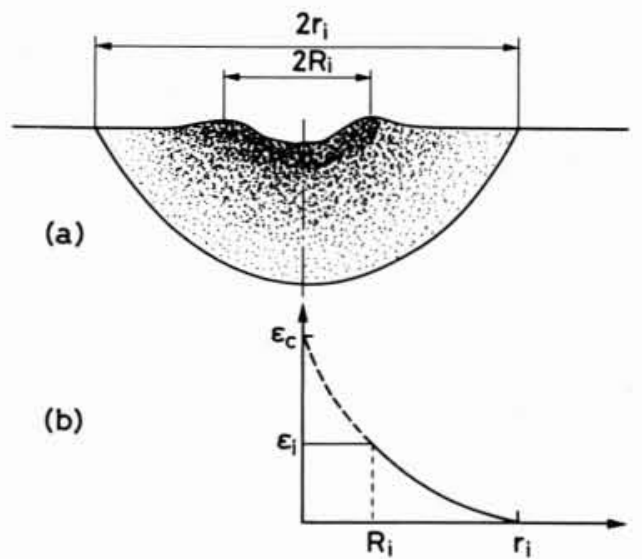

2. Représentation schématique du dégât d'un impact. a) Profil d'un impact; b) Répartition de la déformation en zone d'impact. 


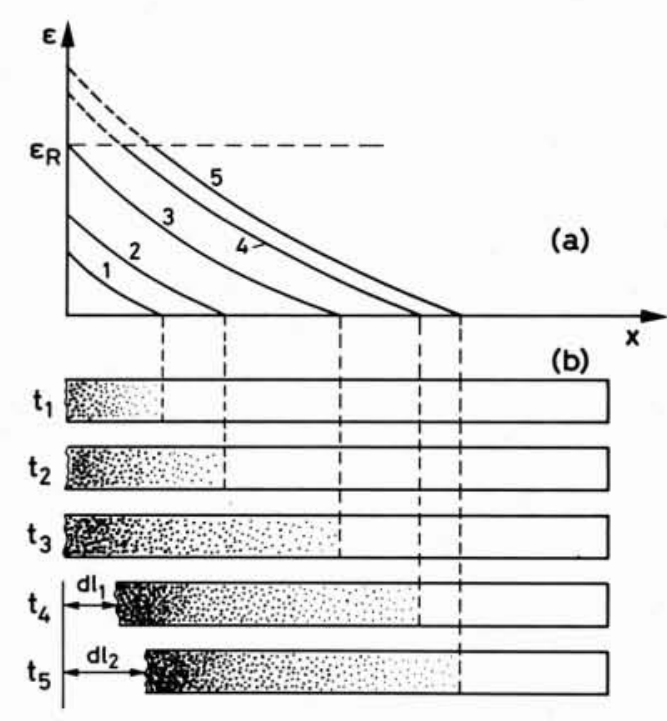

3. Diagramme illustrant la méthode de calcul de la vitesse d'érosion. a) Profils de déformation en différents stades; b) Evolution de l'état de surface et d'écrouissage correspondant.

Pour le générateur du vortex, cette valeur est définie par l'équation (5) :

$$
\sigma_{\mathrm{am}}=\int_{\sigma_{R}}^{\infty} A \sigma^{\alpha} \mathrm{e}^{-\sigma / \beta} \mathrm{d} \sigma / \int_{\sigma_{R}}^{\infty} A \sigma^{\alpha-1} \mathrm{e}^{-\sigma / \beta} \mathrm{d} \sigma .
$$

La combinaison des équations (1), (4) et (5) donne la vitesse d'érosion dans le régime stationnaire,

$$
\begin{aligned}
\dot{E}=\rho S_{m} \dot{N} L\left[\left(\frac{\sigma_{\mathrm{am}}-\sigma_{0}}{\sigma_{R}-\sigma_{0}}\right)^{\frac{1}{n \theta}}-1\right] \times \\
\times\left[1-\int_{0}^{\sigma_{R}} A \sigma^{\alpha-1} \mathrm{e}^{-\sigma / \beta} \mathrm{d} \sigma\right]
\end{aligned}
$$

où $\mathrm{S}_{m}$ et $N$ sont respectivement la taille moyenne et le taux d'impact et caractérisent les paramètres $S(N)$ et $\xi(t)$ dans l'équation (2).

\section{Vitesse d'érosion durant la période d'accélération}

La croissance progressive de la vitesse d'érosion en la période d'accélération est due à deux effets : l'écrouissage et le changement d'état de surface. Au départ, le matériau est relativement ductile et absorbe une partie de l'énergie d'impact en se déformant plastiquement. Au fur et à mesure de l'expérience, il se durcit et l'effet amortissant de déformation "ductile damping » diminue. En conséquence, la partie de l'énergie dissipée par la déformation plastique dans le matériau d'origine est consommée pour augmenter la rupture dans le matériau déjà écroui.
L'effet du changement d'état de surface réside dans la formation d'une zone de concentration de contrainte due aux fractures locales et aux propagations des fissures qui augmentent la probabilité de la rupture et le détachement de la matière et donc la perte postérieure de masse.

Pour calculer l'effet d'écrouissage, on compare le dégât d'un impact sur le même matériau lorsqu'il attaque la surface vierge et lorsqu'il heurte une surface

$$
\begin{array}{r}
w(\text { totale })=w(\text { élastique })+w(\text { plastique })+ \\
+w(\text { rupture }) .
\end{array}
$$

Le calcul peut s'effectuer en utilisant une méthode d'itération. Le résultat final obtenu par le coefficient du durcissement interne $\delta_{\varepsilon}(j)$ après normalisation est :

$$
\delta_{\varepsilon}(j)=\frac{(n+1) \sigma_{0}+\sigma(j)}{(n+1) \sigma_{0}+\sigma_{R}}\left[\frac{\sigma(j)-\sigma_{0}}{\sigma_{R}-\sigma_{0}}\right]^{\frac{1}{n}}
$$

où $n=$ coefficient d'écrouissage du matériau, $j=$ nombre d'itération correspondant au nombre de cycles d'impacts. $\sigma(j)=$ contrainte de déformation après $j$ cycles d'impacts, donnée par la relation suivante :

$$
\begin{aligned}
& \sigma(j)=\frac{1}{n \theta+1} \int_{\sigma(j-1)}^{\sigma_{R}} A \sigma^{\alpha} \mathrm{e}^{-\sigma / \beta} \mathrm{d} \sigma / \int_{\sigma(j-1)}^{\sigma_{R}} A \sigma^{\alpha-1} \times \\
& \times \mathrm{e}^{-\sigma / \beta} \mathrm{d} \sigma .
\end{aligned}
$$

Quant à la quantification du coefficient de changement d'état de surface $\chi_{s}(j)$, nous avons également appliqué la méthode d'itération, en supposant que ce facteur soit proportionnel à la fraction rupture de la surface exposée. La façon la plus simple est alors de calculer le rapport de la surface endommagée à la surface exposée, ceci à la fin de chaque cycle. Les séquences de calcul sont illustrées par les graphes de la figure 4 où l'intensité des impacts est délimitée par les diagrammes $a, d$, $g$ et leur répartition par $b, c, h$. Les bandes sombres dans $c, f$ et $i$ représentent la fraction érodée de la surface exposée à la fin de chaque cycle.
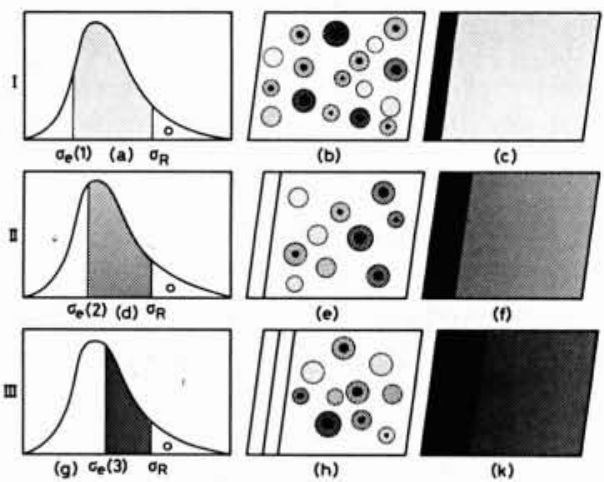

4. Illustration graphique de la méthode de calcul du coefficient de changement d'état de surface. a) Distribution de l'intensité des impacts; $b$ ) Position des impacts sur la surface d'échantillons, les impacts plus intenses étant présentés en plus sombre; c) Fraction érodée de la surface exposée. I, II, III) Situation après un $1^{e r}, 2^{e}$ et $3^{e}$ cycle. 
Par cette méthode de calcul systématique, on obtient de cette manière l'équation suivante :

$$
\chi_{s}(j)=\sum_{k=1}^{j} \frac{1}{\theta^{2}} \frac{1-\mu(R)}{1-\mu_{e}(k)} \prod_{n=1}^{k-1}\left[1-\frac{1}{\theta^{2}} \frac{1-\mu(R)}{1-\mu_{e}(n)}\right]
$$

dans laquelle $\mu_{e}(k)$ est la fraction des impacts dont l'amplitude se situe entre 0 et $\sigma(j)$ et défini dans l'équation (11) :

$$
\mu_{e}(k)=\int_{0}^{\sigma_{1}} A \sigma^{\alpha-1} \mathrm{e}^{-\sigma / \beta} \mathrm{d} \sigma .
$$

Les paramètres $\mu(R)$ et $\mu_{e}(n)$ ont une définition identique à $\mu_{e}(K)$ et correspondent respectivement à $\sigma_{R}$ et $\sigma_{(j-1)}$.

\section{Discussion et application du modèle}

Le présent modèle de prédiction, ayant un caractère déterministe, décrit la vitesse d'érosion comme une fonction explicite des paramètres mécaniques et métallurgiques des matériaux. Ceci facilite considérablement son application pratique par comparaison avec d'autres hypothèses fondamentales empiriques. En effet, l'ensemble des équations peut être résumé dans la relation non dimensionnelle suivante :

$$
\dot{e}=f\left(\sigma_{e}, \sigma_{R}, L, n, \theta\right)
$$

$\sigma_{e}$ et $\sigma_{R}$, respectivement la limite d'élasticité et de rupture sont des paramètres mécaniques. Par contre, $L$, $n$ et $\theta$ sont des paramètres métallurgiques et représentent la profondeur, le coefficient et la puissance d'écrouissage. Ils dépendent tous du mécanisme d'écrouissage et reflètent la réponse des matériaux à l'attaque de cavitation. Ils sont gouvernés en grande partie par l'énergie de faute d'empilement.

Pour tester la validité du modèle, nous l'avons appliqué à un acier inoxydable duplex et à un cuivre pur monocristallin. Les caractéristiques et les propriétés mécaniques de ces alliages sont mentionnés dans d'autres publications. Ici, nous rapportons seulement les résultats (fig. 5). Les lignes continues correspondent aux résultats expérimentaux, tandis que les zones pointillées délimitent les bornes de prédiction du modèle pour différentes divergences dans la précision de mesure des paramètres des matériaux. Comme on constate dans la figure $5 b$, pour un maximum de divergence prévisible, on obtient une déviation de $100 \%-120 \%$ environ. Ceci est dû principalement à la sensibilité du modèle à certains paramètres comme $\theta, L$ et à la simplification faite lors de la description et du calcul. Dans l'état actuel de développement du modèle, ces résultats sont satisfaisants. Les travaux sont en cours afin d'améliorer le concept en introduisant des effets secondaires comme "fatiguechoc » et éventuellement la corrosion. L'aspect phénoménologique du modèle permet son extension et son application aux autres types d'érosion ; par des particules solides ou par l'impact de gouttes du liquide. Il suffira alors d'introduire la fonction de distribution des impacts et des paramètres du matériau relatifs à ces situations dans le programme de calcul.
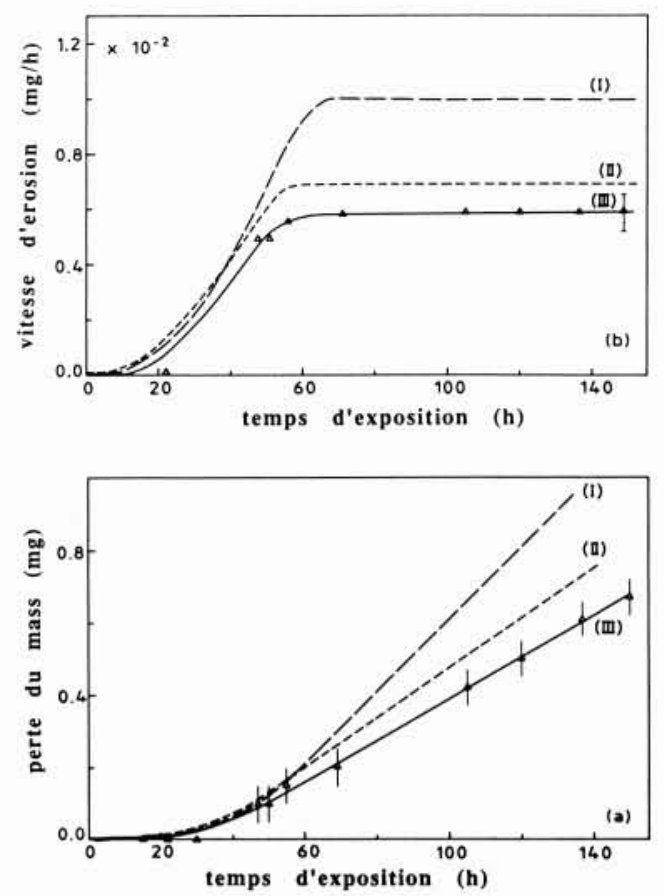

5. Comparaison des courbes d'érosion expérimentale (ligne continue) avec les résultats de prédiction du modèle (zone pointillée) pour l'acier inoxydable duplex et le $\mathrm{Cu}$ pur monocristallin. a) Perte de masse accumulée; b) Vitesse d'érosion en fonction du temps d'exposition.

\section{Références}

J. Asher, A. T. PEACOCK, «A study of disc wear on a pin-disc tester by thin layer activation" in Wear, 81 (1982), pp. $275-284$.

F. Avellan, A. Karimi, «Dynamics of Vortex Cavitation involved in the Erosion of Hydraulic Machines ", in Proc. 7th Int. Conf. on Erosion by Liquid and Solid Impacts, J. E. Field, J. P. Dear, eds., 1987, pp. 25, 1-8.

A. BALL, «On the importance of work hardening in the design of wear resistance materials ", Wear, 91 (1983), pp. 201207.

L. Chincholle, D. Guyomard, « Détection de la cavitation érosive et de l'abrasive ", in Proc. Symp. on hydraulic machinery in the energy related industries, 1980, Tokyo, International Association for Hydraulic Research, vol. 1, pp. $117-130$.

D. J. Finnigan, K. Garbet, I. S. Woolsey, « The application of thin layer surface activation to the study of ErosionCorrosion behaviour " in Corrosion Science, 224 (1982), pp. 359-372.

F. G. Hammitт, «Cavitation Erosion: The state of the art and predicting capability ", in Appl. Mech. Rev., 32 (6), (1979), pp. 665-675.

F. G. Hammitt, Y. C. Huang, C. L. Kling, T. M. Mitchell, L. P. SALOMON, «A statistically verified model for correlating volume loss due to cavitation or liquid impigement ", Characterisation and determination of erosion resistance, ASTM, STP 474 (1970), pp. 288-322.

S. Hattori, B. H. SUn, F. G. Hammitt, « An application of bubble collapse pulse height spectra to venturi cavitation crosion of 1 100-0 Aluminium ", in Wear, 103 (1985), pp. 119-131.

F. J. HeymanN, "On the time dependance of the rate of erosion due to impingement or cavitation ", in Erosion by cavitation or impingement, ASTM, STP 408 (1967), pp. $70-82$. 
F. J. Heymann, «Towards Quantitative Prediction of Liquid Impact Erosion ", in Characterization and Determination of Erosion Resistance, ASTM, STP 474 (1970), pp. 212-248.

F. P. M. H. JANSEn, Proc. 7th Int. Conf. on Erosion by Liquid and Solid Impacts, Cavendish Laboratory, University of Cambridge, J. E. Field, J. P. Dear, eds, 1987, pp. 64, 17.

A. Karimi, F. Avellan, « Comparison of Erosion Mechanisms in Different Types of Cavitation ", in Wear, 113 (3), (1986), pp. 305-322.

A. Karimi, W. R. Leo, «Phenomenological Model for Cavitation Erosion Rate Computation », in Mat. Sci. Eng., 95 (1987), pp. 1-14.

P. V. RAO, C. S. MARTIN, B. C. S. RaO, "Estimation of Cavitation Erosion with Incubation Periods and Materials Properties ", in Journal of Testing and Evaluation, vol. 9, n 3 (1981), pp. 189-197.
B. C. S. RAO, D. H. BUCKLEY, « Deformation and Erosion of f.c.c. Metals and Alloys under Cavitation Attack», in Mat. Sci. Eng., 67 (1984), pp. 55-67.

P. V. RAO, D. H. BuCKLEY, « Predictive Capability of LongTerm Cavitation and Liquid Impingement Erosion Models ", in Wear, 94 (1984), pp. 259-274.

R. Simoneau, N. Désy, R. Grenier, in " Proc. Symp. on Hydraulic machinery, equipment and cavitation ", 1986, Montreal, International Association for Hydraulic Research, vol. 1, 21, pp. 1-7.

A. Thiruvengadam, Proc. 2nd Int. Conf. on Rain Erosion and Allied Phenomena, 1967, Farnborough, U.K. Royal Aircraft Establishment, ed. A. A. Fyall and R. B. King, p. 605 .

S. Vaidya, S. Mahayan, C. M. Preece, Metal. Trans., 11A (1980), pp. 1139-1150.

D. A. WoOdFord, Metal. Trans., 3 (1972), pp. 1137-1145.
Mme NIENALTOWSKA: Si l'on vous fournit un histogramme des impacts et les dimensions de trous dans la phase initiale de l'érosion vous pouvez donc prévoir l'érosion à long terme?

M. KARIMI: En plus de la taille des cratères, il faudra la répartition spatiale et temporelle des impacts dus à la cavitation pour pouvoir prédire le taux d'érosion des matériaux.

M. BAETZ: Je souhaite apporter l'éclairage de l'utilisateur de centaines de roues de turbine. Tout d'abord, grâce au bon dimensionnement, à la bonne conception des profils hydrauliques, à l'enfoncement des machines, adoptés par les constructeurs et EDF, la plupart des roues ne cavitent pas. Toutefois quelquesunes d'entre elles présentent des usures de cavitation qui se produisent pour une durée de 5 à $30000 \mathrm{~h}$.

L'examen des cavitations, dans le parc de nos roues permet de dire qu'il y a similitude des observations avec l'application des propriétés à la fatigue sous eau des matériaux:

- Il existe en effet des seuils de sollicitations de cavitation pour lesquels il n'y a pas d'érosion. Si toutefois un acier est érodé, il est possible par rechargement d'utiliser un acier plus résistant pour lequel l'érosion de cavitation ne se manifestera plus, ou à un degré bien moindre.

Ceci confirme tout à fait les observations de Mme NIENAL TOWSKA sur l'existence d'un seuil de sollicitation donnant lieu à érosion. De même, en fatigue sous eau, il existe pour un nombre de cycles donnés, une valeur de la contrainte pour laquelle il n'y aura pas rupture.

Il existe un temps d'incubation. A l'issue de cette durée, l'aspect de la surface devient mat. De même, en fatigue sous eau, il faut un certain nombre de sollicitations avant de produire les premières fissurations.

De plus, sur le plan pratique, si la surface mat sollicitée en cavitation est repolie, on dispose de nouveau du temps d'incubation et l'on peut de cette manière, éliminer l'action érosive de la cavitation.

De même, en fatigue sous eau, si l'on élimine la fine couche de surface qui a subi l'endommagement et la corrosion, l'on peut repartir pour un cycle complet d'essai.

Ceci permet peut être de dire que pour les niveaux de cavitation auxquels nous sommes confrontés, le métal, sous la surface, n'est pas affecté par l'érosion de surface, sur le plan de ses caractéristiques mécaniques et donc structurales.

J'ai une question à poser : quelle est la nature de la stellite et du matériau appelé « DUPLEX » essayés et pourrions-nous avoir la figure qui a été projetée, représentant le classement de divers matériaux vis-à-vis de la tenue à la cavitation.

M. KARIMI: Le taux d'érosion dépend de l'agressivité de cavitation et de la résistance des matériaux. Pour chaque situation de cavitation, on peut définir une agressivité érosive, par exemple, l'amplitude moyenne des pulses ou l'énergie cinétique des impacts. Pour chaque condition de sollicitation, le matériau présente une limite élastique et une limite de rupture. Maintenant, si l'agressivité érosive de cavitation est inférieure à la limite d'élasticité, il n'y a pratiquement pas d'érosion. Si elle est supérieure a la rupture, l'érosion est très rapide. Lorsque l'agressivité de cavitation se situe entre la limite d'élasticité et la limite de rupture, le taux d'érosion est faible et l'effet de fatigue prépondérant, ce que prévoit notre modèle de prédiction. En effet, en choisissant un matériau plus résistant ou en diminuant la puissance destructive de cavitation, on déplace ces niveaux que je viens de citer dans le bon sens l'un par rapport à l'autre.

Je voudrais quand même préciser que le type de sollicitation dans le cas d'érosion est très différent du cas de fatigue et par conséquent il n'est pas très judicieux de faire une corrélation directe entre le taux d'érosion et l'endommagement dû à la fatigue.

Je pourrai vous donner le type de stellite que nous avons testé et la composition chimique de l'acier DUPLEX ultérieurement.

\section{FINET}

$I^{\text {re }}$ question: Le matériau du "DUPLEX » est-il soudable? Réponse de $M$. KARIMI à M. FINET: pas de problème.

$2^{e}$ question : quand l'énergie est comprise entre $\sigma E$ et $\sigma R$ y a-t-il toujours attaque du matériau?

Réponse de M. KARIMI à M. FINET: Oui.

Même lorsque l'énergie moyenne est comprise entre $\sigma E \sigma R$ il y a évidemment des impacts dont l'amplitude dépasse la limite de la rupture du matériau et qui provoque l'érosion. Dans ces conditions, le taux d'érosion dépend de la position de cette valeur moyenne par rapport à $\sigma E$ et $\sigma R$.

M. COLAS: Dans l'évolution de surface de matériau exposé à la cavitation, il faut aussi considérer les phénomènes de corrosion induits par les écrouissages locaux. Ces écrouissages sont créateurs de niveaux de potentiel différents activant la corrosion et aidant à la décohésion des grains.

Pour une utilisation industrielle le terme de corrosion, constamment réactivé par l'érosion et les écrouissages de cavitation, est très important.

M. KARIMI: C'est exact, l'effet synergique de corrosion-érosion a été vérifié expérimentalement dans plusieurs cas. A l'état actuel, nous n'avons pas encore introduit l'effet de corrosion dans le modèle. Note but a été d'abord d'étudier l'effet mécanique de l'érosion. On peut introduire assez aisément les relations de corrosion sous tension dans des équations d'érosion.

$M$. DOREY : La relativement bonne corrélation entre vos calculs et vos résultats montre que vous avez trouvé les bons paramètres notamment pour le nombre d'impacts. Comment avez-vous fait pour voir les tailles et la puissance des impacts?

M. KARIMI: En ce qui concerne la taille des impacts, on les mesure soit par la méthode de stéréophotométrie utilisant le MEB, soit par une méthode d'interférométrie utilisant un système optique conventionnel.

La puissance des impacts est mesurée à l'aide d'un capteur piézoélectrique miniaturisé. Les signaux des impacts sont ensuite calibrés d'après la dimension des dégâts induits sur des matériaux comme le cuivre ou l'acier duplex. 